\title{
Real-Time Forecasting with EPANET
}

\author{
P. Ingeduld \\ DHI Water \& Environment, Na vrsich 5, 100 00, Prague, Czech Republic; PH (420) \\ 267-227-111; FAX (420) 271-736-912; email: p.ingeduld@ dhigroup.com
}

\begin{abstract}
Automatic on-line simulation provides the system operator with the capability to model the water distribution system in real-time, including predictive estimation of the system behavior. This is essential when performing emergency response and can greatly assist in confirming normal system performance, system trouble-shooting, improvement of system operations, and projection of the current operating scenario. This approach also allows the operator to see how the entire system is operating, instead of relying on the feedback from a few SCADA sensors placed in the network, thus allowing for any breakdown in the network to be readily identified, evaluated, and rectified.

In order to implement the automatic hydraulic simulation decision support tool, which can be used by the water utility operators on real-time basis, the coupling between EPANET 2.0 toolkit and SCADA system has been developed. This coupling operates in the real-time mode and can be used for automatic model update based on the measured data, running periodical steady state simulations, and for forecasting system hydraulics with the next time interval i.e. 3 hours, 1 day, 3 days. Forecasting mode requires proper initial and boundary conditions.

The system initial conditions are retrieved from the SCADA real-time database and the system boundary conditions are developed based on predefined control strategies, historical data, and estimates. The state estimate is needed for node demands and diurnal profiles, water sources, availability of pumps and valves based on the operation and control mode. Additionally, scheduled maintenance and equipment availability needs to be obtained from the SCADA system and included in the automatic setup of the hydraulic model. Forecasted results are transferred to the validation tool, which is used by the system operators, and which allows running corrective simulations. Once validated, the estimated system control variables (setpoints) are uploaded back to the SCADA system and used for automatic scheduling. Similarly, alternate water supply strategies can be quickly modeled and evaluated, guiding the operator until the situation is remedied or stabilizes.

The experience from development and implementation of a system, which performs real-time forecasting of hydraulic behavior of water supply and water distribution systems, is discussed in this paper. The implementation of such system is illustrated on one of the recent case studies.
\end{abstract}

\section{EPANET On-Line}

The concept of EPANET On-Line modeling provides the capability to model the water distribution system in real-time, providing on-line modeling and monitoring 
of the system. This is essential when performing emergency response and it can greatly assist in confirming normal system performance, system trouble-shooting, improvement of system operations, and projection of the current operating scenario. The software can be linked to practically any SCADA monitoring system to operate in one of the operation modes:

- Virtual-Sensor Mode

- Hindcasting Mode

- Event-Simulation Mode

- Predictive Mode

Virtual Sensor Mode receives real time data from the SCADA system and performs an on-line analysis of the system status and response to changing conditions. Based on the state of the system sent on real-time from the SCADA, the use of the pipeline simulation model in real time and under steady state conditions allows computing flows and pressures, for example, even at locations where there are no measurements. The hydraulic, water quality or cost energy model results may be downloaded back into the SCADA database for real time display on the HMI screen.

Hindcasting Mode can model any past event within a defined period (last 1 day, December 12 6am-10am, for example). System history data are retrieved from the SCADA Historical Database. Simulation results can be presented within MIKE URBAN or SCADA interface.

Event-Simulation Mode allows the operator to quickly and easily model the response of the hydraulic system to a number of actions such as start a pump, stop a pump, modification of the flow set points or of the flow demands. Simulation results can be presented within MIKE URBAN or SCADA interface.

Predictive Mode can predict the system behavior within a defined period (next 6,12 hours, 3days, for example). The system initial conditions are retrieved from the SCADA Real-Time database and the system boundary conditions are developed by combining user inputs with the predicted system parameters. The simulated system set-point can be uploaded to SCADA system once the simulation results are validated by the system operator. Validation Simulation results can be presented within MIKE URBAN or SCADA interface.

\section{DIMS (Data Integration and Management System)}

DIMS is used to provide the data exchange between the SCADA real-time or historical database and hydraulic models such as EPANET (DIMS, 2005). Data validation, source data filtering and various gap filling methods are part of the data processing tools. On-line hosts and data drivers are providing the data communication i.e. data reading and writing. DIMS is based on the SQL client-server database and apart from running in the fully automatic and hidden mode it also has quite complex graphical user interface allowing the user to display the measured and modeled data, edit and create measurements, set-up alarms and define reports, and develop VBA macros to control most of DIMS processes. DIMS Web client can optionally be used to provide the user with a limited access via Internet. 


\section{EPANET On-Line}

EPANET Wrapper can work with the standard EPANET2 toolkit DLL library file as well as with the user-developed modifications on that toolkit file. The wrapper receives the real-time data (or other inputs) from DIMS (Data Integration and Management System), makes changes to the template model files (such as EPANET INP file), runs EPANET hydraulic and water quality simulation, and processes the simulation results. All this can run in fully automated and "hidden" or interactive mode.

Most of the system components (Figure 1 EPANET On-Line integration and data diagram) are developed as dynamic libraries, which can be replaced without the necessity to stop the main EPANET On-Line application. This simplifies upgrading the solution to new versions, changing or improving its capabilities, and providing fixes.

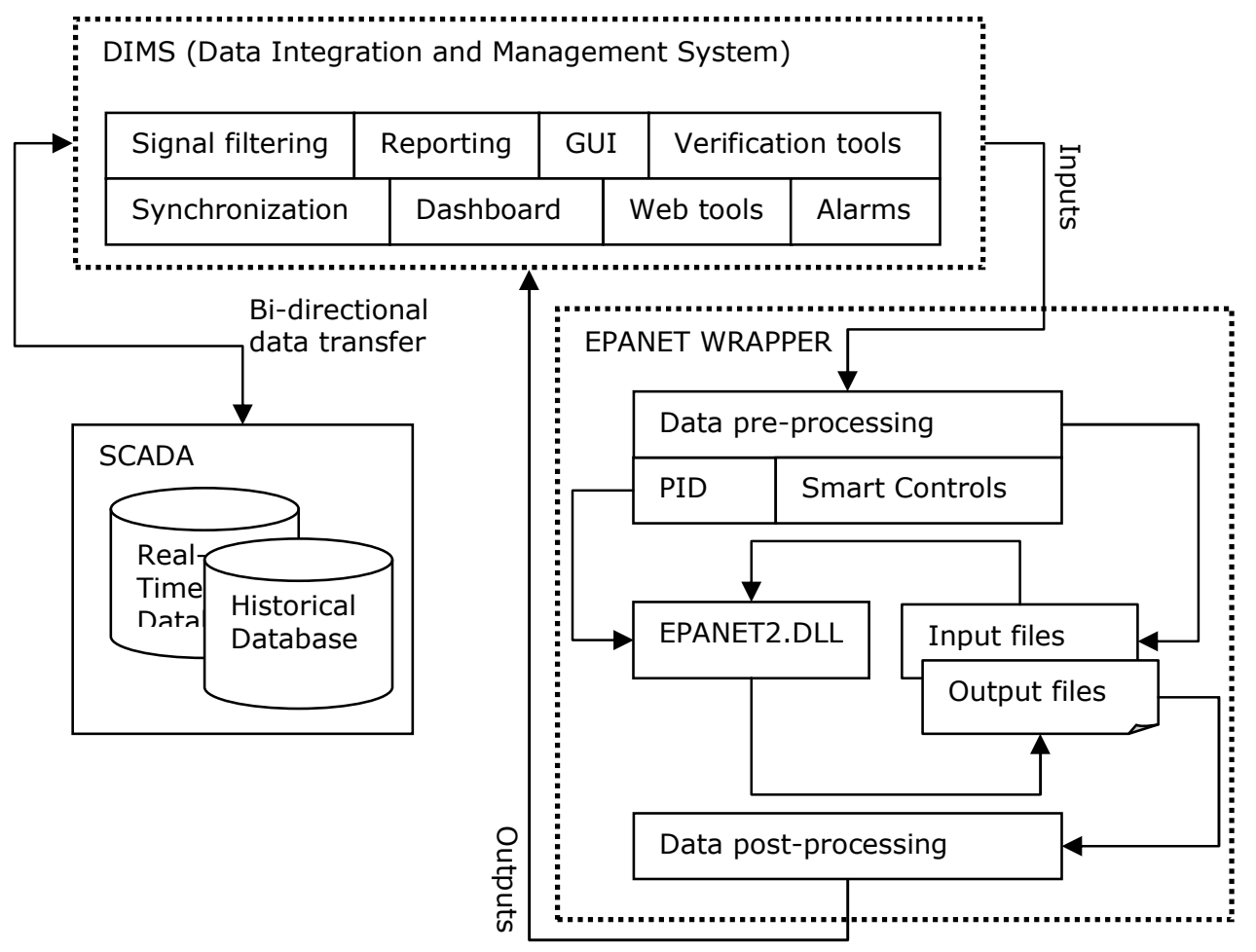

Figure 1. EPANET On-Line integration and data diagram.

The modification of the model template file based on the SCADA data is complex task consisting of several steps. Boundary conditions, such as water levels, pressures, and flow rates are assigned based on the analogue input values. Equipment settings, such as control valve settings, throttle valve openings, pump settings and setpoint values are assigned from analogue input values and inputs such as valve and 
pump statuses are assigned from binary input values. Another step in the input file modification is the demand processing and adjustment. Node demands and diurnal curves are automatically distributed to the junction nodes within each distribution zone, diurnal patterns are scaled and rewound, and all model demands are balanced in order to match the observed zone demands. In this way, it is possible to maintain the mass balance in the model at the same rate as it is measured.

\section{Smart Control}

Controllers on the basis of a pre-programmed operational strategy determine the regulator movements (the control actions). The operational strategy may consist of two parts: the control function(s) and, if more control functions are specified, the control logic (rules), responsible for the selection of an appropriate control function. A control function establishes a relation between a control variable and a controlled variable. A controlled variable can be a regulator setting (e.g. gate position, pump START/STOP level) or some of the flow variables (e.g. water level, flow).

In the latter case, the control decisions are derived by evaluating (comparing) the current value of the controlled flow variable and the pre-defined set-point value. The control algorithm is based on the numerical solution of the "continuous control problem" equation and is usually termed as PID (Proportional-Integral-Differential) control. The actuation signal for the regulator is generated by a PID controller, which usually appears as part of the operational strategy programmed in a Programmable Logical Controller (PLC). At each simulation time step, the set-point is evaluated against the actual value of the control variable (flow or water level, depending on the set-point type). The actual output signal is determined from the following equation:

$$
u=K\left(e+\frac{1}{T_{i}} \int_{0}^{T_{i}} e d t+T_{d} \frac{d e}{d t}\right)
$$

With:

$\mathrm{u}=\quad$ the output signal (i.e. control valve setting, pump speed),

e $=$ the error to be minimized,

$\mathrm{K}=$ the proportionality factor,

$\mathrm{T}_{\mathrm{d}}=$ the derivation time,

$\mathrm{T}_{\mathrm{i}}=$ the integration time.

Apart from PID algorithm, EPANET On-Line allows the user to define and implement his own controls, based on the current time level flows and pressures as well as on any combination of input values such as valve and pump settings, number of operating pumps, storage tank levels and similar.

\section{Demand Forecasting}

Node demand i.e. the water consumption needs to be accurately estimated for the whole duration of the predicted period. There are typically different demand types such as residential, commercial, industry, agriculture, irrigation, leakage depending 
on the type of the water supply or water distribution system to be modeled. Different prediction methods need to be taken into account based on the historical and current consumption data. In simple cases, it is sufficient to base the prediction on the "lastday or last-week" data while in other cases different factors including meteorological parameters needs to be taken into account.

\section{Equipment Availability}

Equipment availability data or maintenance data are very important for the proper function of the Predictive Mode. The modeling system needs not only to obtain the initial state of each equipment such as pump, control valve, tank chamber, and surge tank vessel and such but also the scheduled maintenance data. Time patterns of the equipment availability need to be generated based on the maintenance data within the whole duration of the simulated period.

\section{Set-Points}

The modeling system needs to take the same control decisions as the real control system will make under the same circumstances, such as to start up a number of pumps or adjust a flow set point, and the hydraulic simulator will automatically simulate the results of such control decisions, and in that way predict the behavior of the pipeline system.

Based on the current state of the system, current information on availability of pumps, flow control valves and regulating tanks, and forecasted flow demands, the level set-point values are calculated and, once validated, uploaded to the SCADA system where they will be used for the automatic real-time system control.

\section{Validation of the Plan}

The modeling system predicts flows and pressures within the water supply or distribution network and it calculated the set-points for the automatic SCADA operation. The results of the simulation and the set-point values need to be checked based on different conditions i.e. whether these decisions are sufficient to meet the required demands. If this is not the case, warnings and alarms need to be generated and issued to the system operator informing him on the nature of the problem, e.g. that the level in the storage tank will fall below the low limit tomorrow at 8 a.m. The operator can then revise the demand forecast and re-run the simulation, to see if the problem has been solved by the revision. This process can be repeated until a satisfactory solution has been obtained, Fig 2 . The system operator then validates the revised list, and the new operation plan is sent to the SCADA system. 




Figure 2. Prediction and validation diagram.

\section{Case Studies}

The presented solution has been gradually developed since 1999, when the first component allowing for the real-time modelling was installed and put into an operation. The solution development is mainly driven by the needs of specific implementations for water utilities, which are the primary end users. Some of these implementations are briefly described in the section below.

\section{South-West Moravian Regional Water Supply System, Czech Republic}

The Master Plan Project of South-West Moravian started at the beginning of January 2004. The project involved the development and calibration of the mathematical model of the regional water supply system. The whole regional water supply system (WSS) was divided into two separate models: one for Trebic part (WSS Trebic) and another one for Zdar part (WSS Zdar). Both models were built based on the input data supplied by Zdar and Trebic Water Authorities. The regional water distribution system is supplied from three main and many subsidiary water sources. The main water sources are Water Treatment Plants (WTP) Vir, Mostiste and Stitary. The Zdar's model included 63 storage tanks, 7 main pumping stations and 2 main water sources. The Trebic's model included 50 storage tanks, 10 main pumping stations and 1 main water source.

The main objectives of the modeling works were: (1) Evaluation of normal operating state of the water supply system for existing (year 2002) and future (year 2015) scenarios; (2) Evaluation of water supply system according to quality 
parameters; (3) Optimization of water source improvements and operation of pumping stations; (4) Evaluation of possible profile changes for reconstructed pipes; and (5) Essential precaution at the main distribution system during exceptional conditions, especially during reconstruction of water sources.

The control of the south-west Moravian water supply system is a complicated and complex problem. All the operator's decisions are adopted from the long-term experience of operators in two control rooms - one in WSS Zdar, another one in WSS Trebic. Both WSS are inter-connected and hydraulically dependent. Each control room can have a little different priority from economical point of view. The primary objective of the optimization is to find the optimum system operation for average "usual" conditions, which means the ratio between WTP production, pump scheduling and schedule of operation of the flow valves. The resultant optimal scenario has the following features: (1) Minimum operational costs include the sum of water production costs of all the water sources and energy costs for water pumping; (2) Water level in tanks is between appropriate levels; (3) The water level in tanks at the end of simulation corresponds to the water level at beginning of simulation; (4) It accepts all the technical restrictions such as capacity of pumps, energy limits for pumping stations, water production limits of WTPs, volume of tanks and hydraulic capacity of pipelines. The secondary objective is to keep as high water storage in tanks as possible and to minimize the switching on/off of pumps.

In order to realize optimal performance of the hydraulic and water quality analyses of the water supply system it was decided to link the hydraulic model with the SCADA system. The hydraulic model developed in 'MIKE NET On-Line' receives real time data from the SCADA database (SCADA SCX and Conel) and performs an on-line analysis of the system status based on changing conditions. The model response is compared with the measured response based on the monitoring data to predict the system reliability and also to find system irregularities, including operations problems or changes in consumption patterns.

\section{The Great Man Made River Project, Libya}

The Great Man Made River project is one of the largest civil engineering projects in the world, to be built in five Phases, and designed to provide a dependable and clean water supply to 5 million people. The Phase III of Al Gardabya to Assadaba System links Phase I and II and consists of a $4 \mathrm{~m}$ diameter pipeline with bidirectional flow, two flow control stations, two pump stations and two regulating tanks; installed cost of \$1 US billion.

The hydraulic model of the Phase III transmission pipelines was developed and calibrated using DHI's MIKE NET software. The real-time data collected in the Siemens SCADA system is post-processed in DIMS and used by MIKE NET OnLine for the real-time simulations of flows and pressures. These simulations include a) periodic and automatic model update every 5 minutes and consequent real-time analysis of the actual hydraulic conditions, b) analysis of historical events, and c) prediction of the system behavior for the next 3 and 72 hours.

The EPANET On-Line simulation wrapper was used in order to allow for the implementation of the user-defined complex logic controls, which were used to 
determine the proper settings of regulation valves and pumps. These smart controls are implemented on the iteration level within each time step of the hydraulic solver. The modified (MIKE NET, 2005) EPANET2 engine is included in MIKE NET and MIKE NET On-Line software for both off-line and on-line operation modes. The predictive mode includes different options for estimating the consumption data and set-point variables for the smart controls. The implementation of the On-Line modeling system is done by DHI Group (France, Czech Republic, and Denmark) and Coyne et Bellier (France).

\section{Conclusions}

The presented solution for the automatic on-line simulation provides the system operator with the capability to model the water distribution system in realtime, including predictive estimation of the system behavior. The solution is based on the bi-directional link between the SCADA historical and real-time database and the simulation package, which includes EPANET based hydraulic engine, smart-pre and post-processing and validation and tailored HMI interface used by the system operators.

The solution performs various modeling services including real-time state estimation, hindcasting, and forecasting. It also assists in providing emergency response and in confirming normal system performance, system trouble-shooting, improvement of system operations, and projection of the current operating scenario. This approach also allows the operator to see how the entire system is operating, instead of relying on the feedback from a few SCADA sensors placed in the network, thus allowing for any breakdown in the network to be readily identified, evaluated, and rectified.

\section{References}

Brosnan Thomas M (199) Early Warning Monitoring to Detect Hazardous Events in Water Supplies, An ILSI Risk Science Institute Workshop Report, ISBN: 157881-075-2

Bunn, S, Helms, S (2001) "Application of an expert system to control treated water distribution". Vodafone House, Auckland, New Zealand, Conference Proceedings, New Plymouth District Council, New Plymouth

Cameron, R.W, Barret, R.J., Cazottes, N, Jarrige, P.A, Tocqueville, L (1998) “Link scada with network analysis system: what, why and how?", Proceedings of the Third International Conference on Hydroinformatics, ISBN 905410983 1, Copenhagen, Denmark

DIMS (2005), Software, DHI Water and Environment, Horsholm, Denmark 
EPANET Methodology (1999), Water Supply and Water Resources Division of the U.S. Environmental Protection Agency's National Risk Management Research Laboratory

Farmani, R, Ingeduld, P, Savic,D, Walters, G, Svitak, Z, Berka, J (2005)“'Hydraulic, water quality, and real-time control model of South-West Moravian regional water supply system", Conference proceedings, CCWI, Exeter, UK

Horsner, M, Ingeduld, P (2002) "Real-Time Analysis for Early Warning Monitoring Systems", CIMIC Advanced Technologies in Real-Time Monitoring and Modeling for Drinking Water Safety and Security, Newark, USA

Ingeduld, P: On-Line Analysis with EPANET, Conference Proceedings, 8th annual WD Symposium, EPA Cincinnati, August 27-30, 2006

Ingeduld, P, Maeder, C (2000) Linking Hydraulic Models to Telemetry Systems, Proceedings of 4th International Conference on Hydroinformatics, ISBN, Iowa, USA

MacPhee, M, J (2005) Distribution system water security challenges in the 21st century: a strategic guide, ISBN 1-58321-373-2, AWWA

MIKE NET, MIKE NET On-Line (2005) Software User's Guide, DHI Water and Environment, Horsholm, Denmark 